\title{
A Simple Density with Distance Based Initial Seed Selection Technique for K Means Algorithm
}

\author{
Sajidha Syed Azimuddin ${ }^{1}$ and Kalyani Desikan ${ }^{2}$ \\ ${ }^{1}$ School of Computing Science and Engineering, VIT, Chennai, India \\ ${ }^{2}$ School of Advanced Sciences, VIT, Chennai, India
}

Open issues with respect to $\mathrm{K}$ means algorithm are identifying the number of clusters, initial seed concept selection, clustering tendency, handling empty clusters, identifying outliers etc. In this paper we propose a novel and simple technique considering both density and distance of the concepts in a dataset to identify initial seed concepts for clustering. Many authors have proposed different techniques to identify initial seed concepts; but our method ensures that the initial seed concepts are chosen from different clusters which are to be generated by the clustering solution. The hallmark of our algorithm is that it is a single pass algorithm which does not require any extra parameters to be estimated. Further, our seed concepts are among the actual concepts and not the mean of representative concepts as is the case in many other algorithms. We have implemented our proposed algorithm and compared the results with the interval based technique of Khan. We see that our method outperforms the interval based method. We have also compared our method with the original random $\mathrm{K}$ means and $\mathrm{K}$ Means++ algorithms.

ACM CCS (2012) Classification: Computing methodologies $\rightarrow$ Machine learning $\rightarrow$ Learning paradigms $\rightarrow$ Unsupervised learning $\rightarrow$ Cluster analysis

Information systems $\rightarrow$ Information systems applications $\rightarrow$ Data mining $\rightarrow$ Clustering

Theory of computation $\rightarrow$ Theory and algorithms for application domains $\rightarrow$ Machine learning theory $\rightarrow$ Unsupervised learning and clustering

Keywords: K means, initial seed concept, density, distance, single pass

\section{Introduction}

One of the major demands of various industries is to identify similar patterns of interest. Several variations of clustering algorithms have recently emerged and are successfully applied to real-life data mining problems. A complete clustering algorithm extracts the conceptual differences between the data concepts and assigns every concept into its relevant cluster in order to generate homogeneous concepts within the clusters. Such patterns are required for societal benefits and for the success of implementing various plans in various domains. Some instances which thrust the importance of similar patterns are targeted marketing programs based on customer's base, to estimate similar land usage on earth, to categorize the policy holders based on their insurance claims, for city planning, to identify similar epicenters along continent faults in earthquake studies, in medicine to identify categories of illness conditions and many more. Success of an intelligent clustering algorithm relies on the fact that, with no prior information about the nature of the concepts, homogeneous concepts are to be grouped such that there is high resemblance within the grouped concepts and that there is a significantly low or no resemblance among the inter-clusters. The homogeneously grouped concepts in the clustering solution can be of regular shape (globular) or of arbitrary shape.

Clusters in a clustering solution are separated such that concepts within a cluster are closer to each other than any other concept in other clusters. Clustering solution may be of the following types. They may be center based clusters which are clusters where a concept in a cluster is nearer to the centroid of the cluster (medoid) than to any other centroid of the remaining clus- 
ters. They may be contiguous clusters (nearest neighbor), where a concept in a cluster is more alike to one or more concepts in the cluster than to concepts in other clusters. They may be density based clusters, where high density regions are separated by low density regions, or property based clusters which share the some mutual property or signify a particular concept or may be described by an objective function. Clustering based on partitioning algorithms such as $\mathrm{K}$ means yields fruitful results on mining datasets, when generating a dendrogram representation is infeasible [1]. Our work revolves around Lloyd's [2] K means algorithm. Its significance lies in its simplicity, straightforwardness, and is based on the firm foundation of analysis of variances. It tends to find clusters with approximately the same number of concepts in each cluster, as this method is established on the least squares criterion [3]. The centroids, being the representational units of the clusters, capture the statistical and geometrical meaning of the clustered concepts. The major advantages of this algorithm are that it works easily with any Lp-norm, can be easily parallelized and is insensitive to unordered concepts. This algorithm works well only on concepts with numerical attributes and can be affected negatively by an outlier.

$\mathrm{K}$ means, though known to be one of the best and simplest clustering algorithms, suffers from certain drawbacks. Namely, the algorithm cannot determine the number of clusters, initial seed concepts are randomly selected, it converges to a local minimum, cannot determine the clustering tendency, does not handle empty clusters, cannot identify outliers etc.

We focus on one of the issues mentioned above for $\mathrm{K}$ means - converging to local optimum. This is caused by random selection of initial seed concepts and leads to exhaustive enumeration of all groupings to select the global optimum. Arthur and Vassilvitskii [4] show that the runtime of $\mathrm{K}$ means algorithm is super polynomial. The objective of our work is to generate a globally optimum clustering solution without enumerating all the groupings, by identifying initial concepts such that they are well separated and selected from densely populated regions i.e., they form the representative concepts of different clusters in the solution space, enabling better quality clustering solution.

\section{Related Work}

In the literature many researchers have discussed the importance of identifying the initial seed concepts, which are representative concepts, benefitting in identifying homogeneous groups with high intra-cluster similarity. Astrahan's method [5] uses two parameters, d1 and $\mathrm{d} 2$, where $\mathrm{d} 1$ is used as the radius measure for each point to estimate its density. Then the points are ordered in diminishing order of their densities. The point having the maximum density is selected as the first seed concept. The other seed concepts are selected in the order of diminishing densities with a rule that every new seed concept maintains a minimum distance $\mathrm{d} 2$ away from the previously selected centers. All the points which satisfy these criteria are chosen. If the number of seed concepts obtained is larger than K, they are grouped using hierarchical clustering until only $\mathrm{K}$ of them remains.

Cao et al. [6], streamlined Astrahan's [5] method by a neighborhood-based rough set model. For each concept the $\varepsilon$-neighborhood is identified by estimating the concepts which lie at $\varepsilon$ distance away from it according to a particular distance measure. The first seed concept is selected by sorting the data concepts in non-increasing order of their cohesion and choosing the concept with the maximum cohesion. The subsequent centers are selected by traversing the concepts in non-increasing order of their cohesion and taking the first point having coupling lesser than $\varepsilon$ with the previously chosen centers. The computational cost of $\varepsilon$-neighborhood is lesser than its computational complexity.

Arthur and Vassilvitskii [7] proposed the K Means++ algorithm which solves the seed selection issue by selecting a random concept as the first seed concept and the other seed concepts are selected based on their probable closeness to the previously selected cluster seeds satisfying the triangular inequality such that the farthest seed concepts are selected. Random first seed concept may generate different clustering solutions every time. An extension of $\mathrm{K}$ Means++ is scalable $\mathrm{K}$ Means++ where $\mathrm{K}$ Means ++ is parallelized to increase the speed of this algorithm.

Ostrovsky et al. [8] propose a method to locate seed concepts at positions very close to the op- 
timal centres. It is a 2 stage process, where in stage 1, two initial candidate concepts are picked from the core of the two optimal clusters using a non-uniform sampling process. The remaining candidate concept centers are selected by randomly sampling points with the probability proportional to minimum distance from the previously selected candidate concept centers and are set as the next concept center. This is repeated until $\mathrm{K}$ candidate concept centers are found. In the second step of stage 1, all the given concepts are considered as centres, and then the concept with its corresponding concepts in the Voronoi region are deleted greedily, if they have a minimal contribution towards being selected as centres. Voronoi region concepts for the remaining concepts are re-calculated and the candidate concept centre values are updated. This process is repeated until there are $\mathrm{K}$ centre concepts left along with their corresponding Voronoi region means. The sampling and the deletion procedures are combined to obtain the $\mathrm{K}$ candidate concept centers. In stage 2 , to find the concept centroids there are two approaches. One approach is to use Effros and Schulman [9] method to estimate the radius of the possible clusters around the previously selected candidate concept centres and the mean of the possible clusters gives the final initial seed concepts. The second approach is to expand the Voronoi region of the candidate centre concepts obtained in stage 1 , sample $\frac{4}{\beta \omega}$ random concepts from the Voronoi region by fixing parameter $\beta$ and an input parameter $\omega$. Final centres are selected by exhaustively searching for the $\mathrm{K}$ candidate concepts (choosing one candidate per initial centre from the samples of each Voronoi region) which are closer to the mean and form the final seed concepts.

Khan [10] locates the seed concepts by uncovering the highest gaps in the dataset, extracting the cluster boundaries and figuring out the mean of the concepts within the cluster boundaries. This method performs better than $\mathrm{K}$ Means++ [7] only in few cases.

The Single Pass Seed Selection algorithm proposed by Karteeka Pavan et al. [11], identifies initial seed concepts using a single pass algorithm by taking the initial seed concept as the concept with maximum density and ensuring that the remaining concepts selected are far- thest away from the previously selected concepts. Only the density of the initial seed point is considered and the remaining seed concepts are only selected based on distance from the previous seed concepts. In this method, it has been assumed that there is an equal number of concepts in every cluster, which may not always be true.

A heuristic approach to find the number of clusters and initial seed point was proposed by Vighnesh Birodkar and Reddy [12]. The first seed point selected is random and then the remainder is based on measuring the farthest point from the previously selected seed concepts. It uses a heuristic to terminate the seed selection process. Also, it assumes that the clusters are well separated in a certain way, so it cannot be applied in all the cases.

Chaudhuri and Chaudhuri [13], identify (multivariate) non-elliptical or elongated clusters which require more than a single seed point by a border point detection technique. The initial seeds are selected from a densely populated region and a spanning tree method is used to assign several seed concepts to a cluster once it is identified as an elongated cluster.

Md Anisur Rahman et al. [14] produce a set of high quality initial seeds by a Seed-Detective algorithm which uses ModEx technique to generate preliminary clusters applying decision tree technique. A large amount of memory is utilized to find out the intersection of the leaf-nodes of the decision trees. The seeds for numerical attributes are obtained by finding the mean of the numerical values from the preliminary clusters and the seeds for categorical attributes are found by selecting the highest frequency records from the preliminary clusters. These are then fed as input to $\mathrm{K}$ means to generate the final clusters.

Mengqiu Tian et al. [15] make use of a histogram analysis technique to automatically initialize the $\mathrm{K}$ means algorithm. They overcome the inaccurate segmentation that arises due to data acquisition and reconstruction of the artifacts of CT images which have several contents with similar gray-levels. Here the key idea is to spot the peaks in histograms and the graylevel of the maximum peak in the histogram is selected as the first eligible centroid. The subsequent centroid chosen is the local maxi- 
mum having the highest weighted distance of all other known centroids (Viterbi algorithm). One of the findings of this algorithm is that the segmentation should not only be based on gray levels, but should also take into consideration all the attributes or spatial information.

Scott Epter et al. [16] discuss a graph based system for detecting clusterability of the concepts and generating seed information, including an estimate of the value of K. A histogram is constructed by estimating the bucket size from the distance matrix and plotting the distances in the histogram from which it may be identified if the concepts are clusterable or not. Based on the separation of the peak concepts and if well separable concepts are identified, the seed concepts can be estimated from connected lines of the histogram. This technique works well only for well separated groups of concepts.

Md Anisur Rahman et al. [17] consider only the density of each data concept (mixed data concepts) while identifying the initial seed concepts and ignoring the distance between them.

Some of the researchers, Astrahan [5], Cao et $a l$. [6] focus on identifying seed concepts based on certain parameters. Astrahan's method [5] depends on parameter $\mathrm{d} 1$, which is the radius of every concept to be considered for density estimation and parameter $\mathrm{d} 2$, which is the minimum distance to be satisfied from the previous seed points; whereas the method of Cao et al. [6] depends on the set of concepts within $\varepsilon$ distance from the selected seed concept based on a particular distance measure. Arthur and Vassilvitskii [7] ensure that the seed concepts are far away from each other, but do not guarantee reliability of the solution due to randomness involved or, is successful in identifying the seed concepts but leads to over splitting of the clusters as in Khan [10]. Density of the first concept is considered by Karteeka Pavan et al. [11] but the remaining are selected based on only distance from the previous concepts, whereas Mengqiu Tian et al. [15], Scott Epter et al. [16] use histogram based approaches which perform well on only well separated clusters.

The main contributions of this paper are as follows. We propose a density based seed selection technique which takes into account both density and distance from the previously selected seed concepts of all the data concepts.
Selection is based on the criteria that higher weightage is given to distance than to density so that the seed concepts are well separated and also selected from highly dense regions. Our proposed work ensures that no other estimation is needed in identifying the seed concepts. One of the major drawbacks of the previous approaches is that when outliers are present in the data, their contribution in the seed selection will cause a huge deviation in the selected concepts, making the clustering of the concepts harder. Our method ensures that such a concept is not selected as we determine the seed concepts by prioritizing based on the criteria of density with distance from the previous seed concepts. Our proposed algorithm is a single pass algorithm as the distances between all the concepts are maintained in a distance table, and are reused while calculating the distance of all the concepts from the previously selected seed concepts. We have implemented our proposed technique and compared the results with Khan's method [10], which is interval based, random $\mathrm{K}$ means [2] and K Means++ method [7] (20 iterations) and proved that our method outperforms or performs on par with the interval based and other methods.

\section{Proposed Seed Selection Method}

We propose a density with distance based method which is a simple method to administer. Its highlight is that its working takes into account both the distance between the seed concepts and their corresponding density. Our proposed method overcomes the limitations of the previous approaches by locating seed concepts which are farthest from the previously selected seed concepts, ensuring that they represent the clusters and are in the densest regions, by giving a higher weightage for the distance between the concepts and lowering the weightage for density. This ensures that the selected seed concepts are guaranteed to be selected from dense regions of concepts which are well separated from each other. Here no additional estimation needs to be made in identifying the seed concepts. Our method guarantees that the initial seed concepts are taken from different clusters that are to be generated by the clustering solution. When these concepts are given as the 
Step 1. For each data concept $C_{i}, i:=1 \ldots n$

For each data concept $C_{j}, j:=1 \ldots n$
$\quad$ Calculate $E D_{i j}:=\operatorname{dist}\left(C_{i}, C_{j}\right)$ where $\operatorname{dist}\left(C_{i}, C_{j}\right):=\sqrt{\left(C_{i}-C_{j}\right)^{2}}$.

Step 2. For each data concept $C_{i}, i:=1 \ldots n$

Compute Row-Sum $R S_{i}=\sum_{j=1}^{n} E D_{i j}$.

Step 3. Sort Row-Sums $\left(R S_{i}\right)$ with the corresponding indices of the concepts in non-decreasing order to get the Sorted Row-Sum (SRS).

Step 4. Find the index of the first seed point $I S C_{1}:=$ index_of_concept $[\min (S R S)]$

Step 5. For 1: $=2$. . K

Step 5.1. For $i:=1 . . n$

$$
\begin{aligned}
& \text { Step 5.1.1. For } j=1 . l-1 \\
& \text { Get } g_{i j}:=E D\left[i, I S C_{j}\right] \\
& \text { Step 5.1.2. Get } S_{i}:=\min \left(g_{i j}\right)
\end{aligned}
$$

Step 5.2. Sort $S_{i}$ with the corresponding indices of the concepts in non-increasing order to get the Sorted Distance Values $(S D V)$

Step 5.3. With $\alpha$ : $=0.6$ or 0.8

For each data concept $C_{i}, i:=1 . . n$

New_Den_Dist_Index $(N D D I):=\alpha *\left(\right.$ index of $C_{i}$ from $\left.S D V\right)+(1-\alpha) *\left(\right.$ index of $C_{i}$ from $\left.S R S\right)$

Step 5.4. Obtain the index of the minimum value in $N D D I$

ind $N D D I:=$ index $[\min (N D D I)]$

$I S \overline{C_{1}}:=$ ind $N D D I$

Step 6. Retrieve the seed concepts (Act_Seed) using their index values in $I S C_{1}, I S C_{2}, \ldots, I S C_{K}$

Step 7. Cluster the data concepts using the seed concepts in Act_Seed.

Figure 1. Proposed seed selection algorithm.

initial concepts for clustering to $\mathrm{K}$ means algorithm, the clustering solution obtained ensures a global optimum solution of high quality.

Our proposed algorithm is as given in Figure 1. In step 5.3 we assign a value $\alpha:=0.6$ or 0.8 for the distance index which ensures that the seed concepts selected are well separated. Step 5.4 ensures that we select a concept from a dense region which is placed farthest from the previously selected concepts. This confirms that the seed concepts are the representative points of the clusters. The proposed methodology guarantees that the first seed concept is selected from a dense region and the remaining concepts are prioritized based on density, simultaneously ensuring that they are at a maximum distance from the previously selected seed concepts. This list is updated until $\mathrm{K}$ seed concepts are identified.

Our seed concepts are among the actual concepts, whereas most of the existing methods capture the seed concepts as the mean of the initial set up which are representative concepts. Since during first iteration of the clustering process, the proposed method compares all the concepts with the seed concepts which are actual concepts and are the representative points of each cluster, they bring together similar concepts which are identical to each other. The time complexity of our proposed algorithm is observed as $O\left(|C|^{2}\right)$, where $|C|$ is the total number of data concepts.

\section{Experimental Results and Discussion}

All the experimental datasets were normalised using Min-Max normalisation given by the formula

$$
Z_{i}=\frac{\left[C_{i}-\min (C)\right]}{[\max (C)-\min (C)]}
$$

where $C:=\left\{C_{1}, \ldots, C_{n}\right\}$ are the given concepts to be clustered and $Z_{i}$ is the $i$-th normalized data. The normalized data lies between $[0,1]$. 
To ensure that the cluster assignments are replicable using this methodology, clustering was performed on the same data many times. For each of the runs, no change was observed in the cluster centres. All experiments were conducted on a HP laptop with an Intel (R) Core (TM) i-55200U CPU@2.20GHz Pentium processor and $8 \mathrm{~GB}$ of main memory with 64bit OS.We carried out our experiment on 7 datasets and compared our results with the interval based method, $\mathrm{K}$ means and $\mathrm{K}$ Means++ algorithms. The first dataset that we considered was the standard Iris [18] dataset. Using the petal length attribute, it was partitioned into 3 clusters. Cloud Cover data is the second dataset downloaded from Phillipe Collard [19]. Fifty partitions were generated from 1024 concepts. Data of attribute 3 was used to perform the clustering. Abalone dataset from UCIMLR [20] was the third dataset. Data in column 5 was used for clustering 4177 instances into 25 groups. The fourth dataset was Ionosphere from UCIR. Using the data from column 5, 351 concepts were clustered into 2 partitions. Wine dataset is the fifth dataset with 179 concepts clustered into 3 groups using the data in column 13-Proline. The sixth dataset was Glass with the data in column 3-RI: refractive index clustered into 2 partitions (Window glass-163, Non-window glass-51). The last was a synthetic dataset of 94 concepts with non-uniform distribution grouped into 5 and 7 clusters.

The interval based method focuses on identifying gaps between data concepts and ensuring that the seed concepts are closer to the mean. This leads to subdivision of dense regions as shown below in Figures 2 and 3. We have randomly generated 94 synthetic data concepts which are non-uniformly distributed (range of values: $1-120$ ). We clustered them into 5 and 7 clusters. Figure 2 and Figure 3 show the comparative plots for 5 and 7 clusters for the proposed method with $\alpha=0.6$ and 0.8 (both SSE values are same) and the interval based method.

In Figure 2 we see that the interval based method captures the largest gaps between the concepts and provides seed values based on the gaps which lead to oversplitting, whereas using our method the clusters are separated based on density of the regions.

We can also see in Figure 2 that our proposed method of clustering into 5 clusters with $\alpha=0.6$

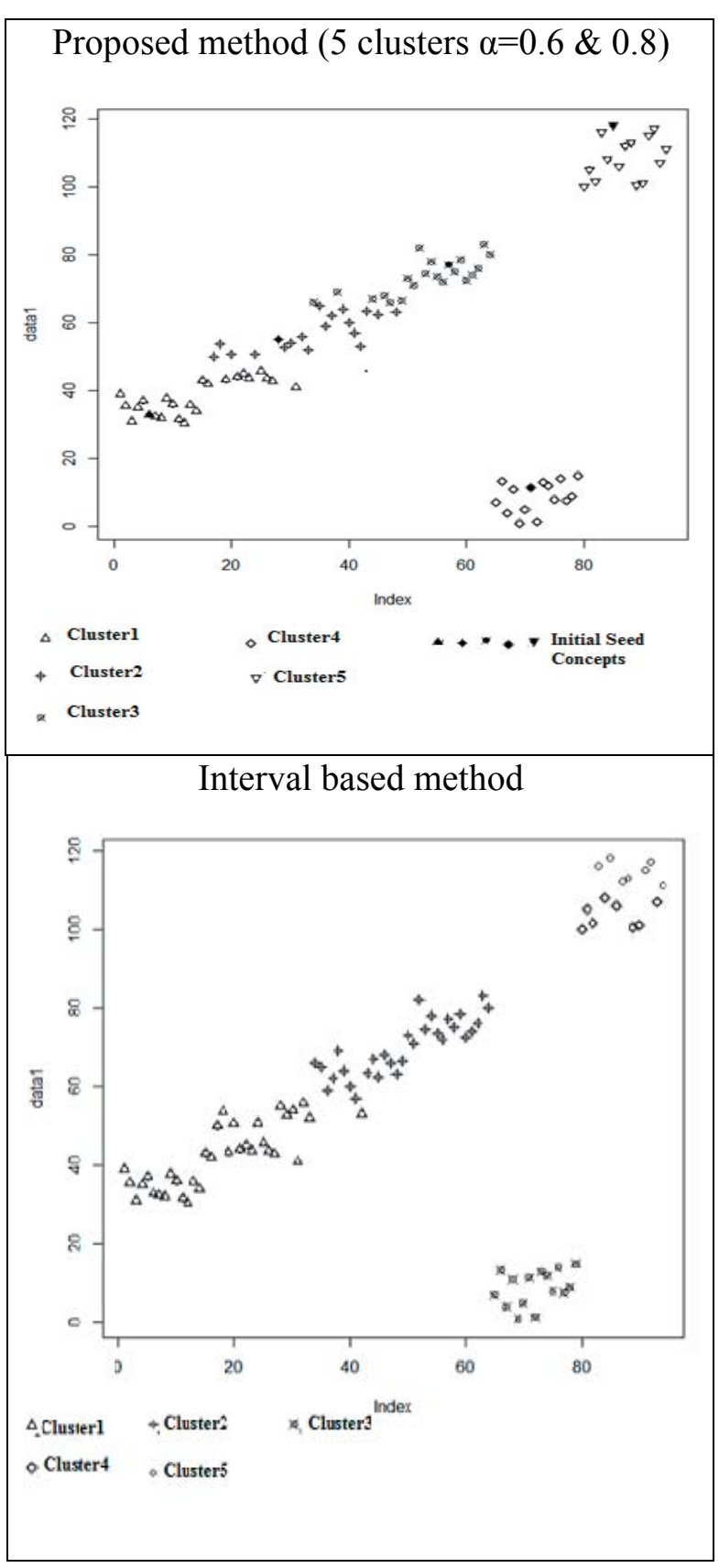

Figure 2. Comparative plot for synthetic data concepts (5 clusters).

was able to identify clusters from ranges $30-$ $40,40-65,60-80$ as cluster 1 , cluster 2 , cluster 3 and $1-15$ as cluster 4 and $100-120$ as cluster 5 of sizes $22,15,15,18,24$ respectively. In interval based method, concepts which are assigned to cluster 5 in our method are assigned to two different clusters of sizes 8 and 7 , as this method focuses only on the distance between two adjacent concepts, ignoring the density. This leads to oversplitting of homogeneous 
regions. The other clusters obtained by this method are of sizes 15, 34 and 30 respectively.

Figure 3 shows the clustering for 7 clusters. Values from $20-30$ were in cluster $1,40-50$ in cluster $2,50-60$ in cluster $3,60-70$ in cluster 4, $80-90$ in cluster 5,100-120 in cluster 6 and $1-15$ in cluster 7 of sizes $11,15,15,15,13,14$, 11 , respectively. In interval based method, we see the difference in cluster formation in cluster

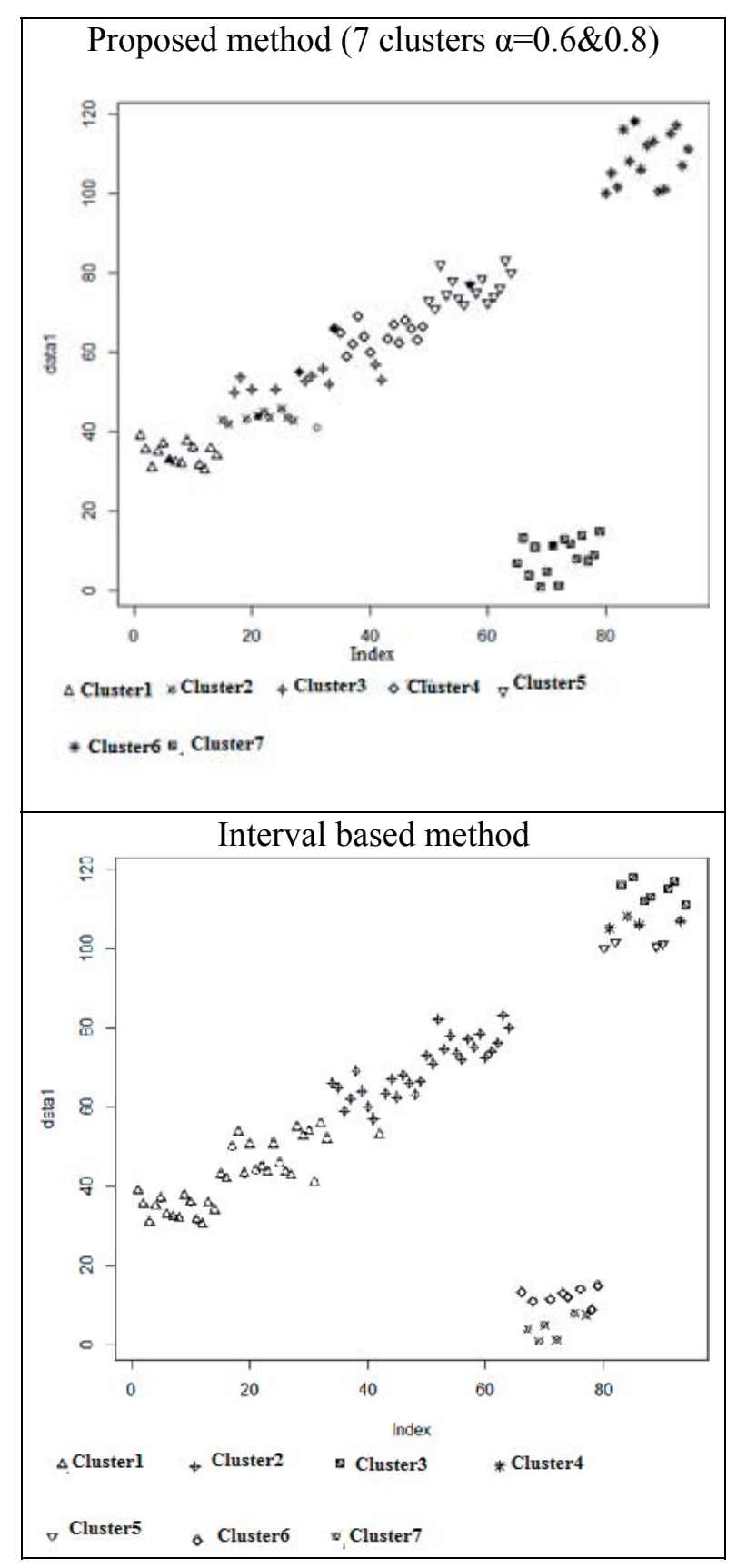

Figure 3. Comparative plot for Synthetic data concepts (7 clusters).
$6(100-120)$, which is subdivided into 3 clusters of sizes 4, 4, 7 and cluster $7(1-15)$ divided into 2 subclusters of sizes 7, 7 with other clusters of sizes 34 and 30 . This situation arises as gaps are considered during seed identification.

We estimated the accuracy of the clustering solution by dividing the sum of intra-cluster similarity by the total number of concepts (SSE Normalised). The Sum of Squared Errors or Residual Sum of Squares [21] of a clustering solution is used to estimate the tightness of the solution obtained. The smaller the value, the purer is the solution and a value 0 indicates that the cluster is in its purest form.

We validated the accuracy of clustering solution using the Sum of squared differences between cluster members and their closest centres (normalized by data size) as a measure of comparison as mentioned in [10].

Table 1 presents the reduction percentage of SSE (Sum of squared differences between cluster members and their closest centres (normalized by data size)) of our proposed method with bias parameter $\alpha$ as 0.6 or 0.8 respectively, based on the best SSE values observed vis-avis the interval based method, random $\mathrm{K}$ means and $\mathrm{K}$ Means++ (20 trials). From the results obtained, we see that for cloud cover, abalone, ionosphere, wine and synthetic data concepts the proposed method outperforms the interval based method. Also, we see that, except for wine data concepts, the proposed method outperforms or performs similar to random $\mathrm{K}$ means. Finally, we observe that, excluding cloud cover dataset, the proposed method outperforms or performs on par with $\mathrm{K}$ Means++.

Table 2 presents the total time taken in seconds to identify the seeds and perform the clustering. For example, if we consider the Iris dataset, the total time taken for our proposed method is $0.69 \mathrm{sec}(0.63 \mathrm{sec}$ (seed selection) $+0.03 \mathrm{sec}$ (Clustering time)) when $\alpha=0.6$. This time, as can be seen in Table 2, is higher than the execution time for interval based method and $\mathrm{K}$ Means++. This is expected as our proposed algorithm works on the principle of identifying the seed concepts from a dense region and parallelly ensuring that the seed concepts are well separated. No other additional computation is done in the proposed algorithm. The other approaches do not ensure that the seed concepts 
Table 1. Reduction\% of SSE (normalized) of proposed method vis-à-vis interval based method,

Random K means, K Means++ (20 trials).

\begin{tabular}{|c|c|c|c|c|c|c|c|c|c|}
\hline \multirow[b]{2}{*}{ Dataset } & \multirow[b]{2}{*}{ Clusters } & \multicolumn{2}{|c|}{ Proposed method } & \multirow{2}{*}{\begin{tabular}{|c|} 
Interval \\
based \\
method SSE
\end{tabular}} & \multirow{2}{*}{$\begin{array}{c}\text { Reduction\% } \\
\text { of SSE with } \\
\text { Interval } \\
\text { based }\end{array}$} & \multirow[b]{2}{*}{$\begin{array}{l}\text { Random K } \\
\text { means SSE }\end{array}$} & \multirow{2}{*}{$\begin{array}{c}\text { Reduction\% } \\
\text { of SSE with } \\
\text { random K } \\
\text { Means }\end{array}$} & \multirow{2}{*}{$\begin{array}{c}\text { K means }++ \\
(20 \text { trials }) \\
\text { SSE }\end{array}$} & \multirow{2}{*}{$\begin{array}{c}\text { Reduction \% } \\
\text { of SSE } \\
\text { with K } \\
\text { Means }++ \\
\end{array}$} \\
\hline & & \begin{tabular}{|c|}
$\begin{array}{c}\text { Best } \\
\text { when } \\
\alpha \\
\end{array}$ \\
\end{tabular} & SSE & & & & & & \\
\hline Iris & 3 & 0.6 & 0.004761141 & 0.004761141 & $0 \%$ & 0.004761141 & $0 \%$ & 0.00484622 & $1 \%$ \\
\hline $\begin{array}{l}\text { Cloud } \\
\text { Cover }\end{array}$ & 50 & 0.8 & 0.00003522 & 0.00005123 & $31 \%$ & 0.00003865 & $9 \%$ & 0.00002906 & $-21 \%$ \\
\hline Abalone & 25 & 0.8 & 0.000101299 & 0.00015423 & $34 \%$ & 0.0001050 & $4 \%$ & 0.0001412 & $28 \%$ \\
\hline Ionosphere & 2 & 0.6 & 0.01701439 & 0.02604707 & $35 \%$ & 0.0680575 & $75 \%$ & 0.0170143 & $0 \%$ \\
\hline Wine & 3 & \begin{tabular}{|c|}
$0.6 \&$ \\
0.8
\end{tabular} & 0.0074268 & 0.00763966 & $2 \%$ & 0.00668192 & $-11 \%$ & 0.0076396 & $2 \%$ \\
\hline Glass & 2 & 0.6 & 0.009820371 & 0.00982841 & $0 \%$ & 0.00983212 & $0 \%$ & 0.0098284 & $0 \%$ \\
\hline \multirow{2}{*}{$\begin{array}{c}\text { Synthetic } \\
\text { data }\end{array}$} & 5 & $\begin{array}{c}0.6 \& \\
0.8\end{array}$ & 0.001903122 & 0.00310363 & $39 \%$ & 0.00190359 & $0 \%$ & 0.0019237 & $1 \%$ \\
\hline & 7 & $\left|\begin{array}{c}0.6 \& \\
0.8\end{array}\right|$ & 0.001009916 & 0.00289865 & $65 \%$ & 0.00101038 & $0 \%$ & 0.0010099 & $0 \%$ \\
\hline
\end{tabular}

Table 2. Comparison of time of execution in seconds of proposed method vis-à-vis interval based method, Random K means, K Means++ (20 trials).

\begin{tabular}{|c||c|c|c|c|c|}
\hline Dataset & Clusters & Best $\boldsymbol{\alpha}$ & $\begin{array}{c}\text { Proposed } \\
\text { method }\end{array}$ & $\begin{array}{c}\text { Interval based } \\
\text { method }\end{array}$ & K means++ (20 trials) SSE \\
\hline \hline Iris & 3 & $\mathbf{0 . 6}$ & 0.69 & 0.05 & 0.04 \\
\hline Cloud Cover & 50 & $\mathbf{0 . 8}$ & 3.11 & 0.44 & 1.89 \\
\hline Abalone & 25 & $\mathbf{0 . 8}$ & 18.03 & 2.74 & 3.04 \\
\hline Ionosphere & 2 & $\mathbf{0 . 6}$ & 1.24 & 0.08 & 0.07 \\
\hline Wine & 3 & $\mathbf{0 . 6} \& \mathbf{~ 0 . 8}$ & 1.49 & 0.08 & 0.07 \\
\hline Glass & 2 & $\mathbf{0 . 6}$ & 1.51 & 0.07 & 0.08 \\
\hline \multirow{2}{*}{ Synthetic data } & 5 & $\mathbf{0 . 6} \& \mathbf{~ 0 . 8}$ & 1.47 & 0.56 & 0.08 \\
\cline { 2 - 6 } & 7 & $\mathbf{0 . 6 ~ \& ~ 0 . 8}$ & 2.09 & 1.05 & 0.09 \\
\hline
\end{tabular}

are selected from dense regions and are only distance based. Hence, the time taken by those algorithms is less. Nevertheless, our proposed approach ensures increased accuracy in selecting the seed concepts. Thus, the seed concepts identified using our method ensure good quality clusters, for most of the data sets.

\section{Conclusion}

In this study we considered 7 datasets for our analysis. For each of these 7 datasets, our proposed method was compared with interval based method, $\mathrm{K}$ means and $\mathrm{K}$ Means++ algorithms. The largest of the datasets considered by us is Abalone dataset with 4177 instances and the smallest dataset is the Iris dataset with 150 instances. For each of the datasets, clustering solutions were obtained by considering the most contributing attribute.

The central objective of our proposed algorithm is to yield replicable results for the given dataset with high clustering accuracy. The sums of squared differences between cluster concepts and cluster centres obtained using our proposed method and the interval based method, random $\mathrm{K}$ means and $\mathrm{K}$ Means++ were compared and presented in Table 1. Our proposed method outperforms interval based method for almost all the datasets and is on par for Iris dataset. With respect to $\mathrm{K}$ Means++, our method improves the results for some datasets, whereas for cloud 
cover dataset our method underperforms. We infer from Table 2 that our proposed method takes more time compared to other approaches, due to the fact that our approach takes into consideration both density and distance during seed selection. Albeit, our approach yields better or equivalent clustering solutions compared to the existing approaches.

\section{Acknowledgements}

The authors are very grateful to the anonymous reviewers and editor. Their many helpful and constructive comments and suggestions have helped us to significantly improve this work.

\section{References}

[1] A. K. Jain et al., "Data Clustering: A Review", ACM Computing Surveys, vol. 31, no. 3, 1999. http://dx.doi.org/10.1145/331499.331504

[2] Stuart P. Lloyd, "Least Squares Quantization in PCM", IEEE Transactions on Information Theory, vol. 28, no. 2, pp. 129 - 136, 1982. http://dx.doi.org/10.1109/TIT.1982.1056489

[3] W. S. Sarle, "Cluster Analysis by Least Squares", in Proceedings of the Seventh Annual SAS Users Group International Conference, Cary, NC: SAS Institute Inc, pp. $651-653$.

[4] D. Arthur and S.Vassilvitskii, "How Slow is the K-Means Method?", in Proceedings of the twenty-second annual symposium on computational geometry, ACM Press, 2006.

http://dx.doi.org/10.1145/1137856.1137880

[5] M. M. Astrahan, "Speech Analysis by Clustering or the Hyperphoneme Method", Tech. Rep. AIM124, Stanford University, 1970.

[6] F. Cao et al., "An Initialization Method for the K-Means Algorithm Using Neighborhood Model", Computers and Mathematics with Applications, vol. 58, no. 3, pp. $474-483,2009$. https://doi.org/10.1016/j.camwa.2009.04.017

[7] D. Arthur and S.Vassilvitskii, "K-means++: The Advantages of Careful Seeding", in $S O D A$, pp. 1027 - 1035, 2007.

[8] R. Ostrovsky et al., "The Effectiveness of LloydType Methods for the K-Means Problem", in Symposium on Foundations of Computer Science, 2006. http://dx.doi.org/10.1145/2395116.2395117

[9] M. Effros and L. J. Schulman, "Deterministic Clustering with Data Nets", in Proc. ISIT, 2004.
[10] F. Khan, "An Initial Seed Selection Algorithm for K-Means Clustering of Geo-Referenced Data to Improve Replicability of Cluster Assignments for Mapping Application", Applied Soft Computing, vol. 12, no. 11, pp. $3698-3700,2012$. https://doi.org/10.1016/j.asoc.2012.07.021

[11] K. K. Pavan et al., "Single Pass Seed Selection Algorithm for K-Means", Science Publications Journal of Computer Science, vol. 6, no. 1, pp. $60-66,2010$.

[12] V. Birodkar and D. R. Edla, "Enhanced K-Means Clustering Algorithm using A Heuristic Approach", Journal of Information and Computing Science, vol. 9, no. 4, pp. 277 - 284, 2014.

[13] D. Chaudhuri and B. B. Chaudhuri, "A Novel Multiseed Nonhierarchical Data Clustering Technique", IEEE Transactions on Systems, Man, and Cybernetics - Part B: Cybernetics, vol. 27, no. 5, 1997. http://dx.doi.org/10.1109/3477.623240

[14] Md. A. Rahman et al., "ModEx and Seed-Detective: Two Novel Techniques for High Quality Clustering by using Good Initial Seeds in K-Means", Journal of King Saud University Computer and Information Sciences, pp. 93-236, 2015.

https://doi.org/10.1016/j.jksuci.2014.04.002

[15] M. Tian et al., "An Automatic Histogram-based Initializing Algorithm for K-Means Clustering in CT", in Proceedings des Workshops Bildverarbeitung für die Medizin, Heidelberg, Germany, pp. $277-282,2013$.

[16] S. Epter et al., "Detection and Initial Seed Selection in Large Data Sets", SIAM Workshop on Clustering High Dimensional Data and its Applications, Arlington, (with SIAM data mining conference), April 2002.

[17] Md. A. Rahman et al., "DenClust: A Density Based Seed Selection Approach for K-Means", ICAISC: Artificial Intelligence and Soft Computing, pp. 784 - 795, 2014.

[18] R. A. Fisher, "Iris Data Set", in: R. A. Fisher (Ed.), UC Irvine Machine Learning Repository, 1936. http://archive.ics.uci.edu/ml/machine-learningdatabases/iris/iris.data

[19] P. Collard, "Philippe Collard's Cloud Cover Data", 1989.

ftp://ftp.ics.uci.edu/pub/machine-learning-databases/undocumented/taylor/cloud.data

[20] W. J. Nash et al., "Abalone Data Set", in: S. F. Division (Ed.), UC Irvine Machine Learning Repository, 1994.

http://archive.ics.uci.edu/ml/machine-learningdatabases/abalone/abalone.data

[21] C. D. Manning et al., "Introduction to Information Retrieval", Cambridge University Press, 2008. https://www-nlp.stanford.edu/IR-book/ 
Received: April 2017

Revised: October 2017

Accepted: October 2017

Contact addresses:

Sajidha Syed Azimuddin

School of computing sicence and engineerg

VIT, Chennai

India

e-mail: sajidha.sa@vit.ac.in

Kalyani Desikan

Department of mathematics School of Advanced Sciences

VIT, Chennai

India

e-mail: kalyanidesikan@vit.ac.in
SAJidHa Syed AzImUdDin is a selection grade assistant professor in the School of Computing Science and Engineering, VIT, Chennai, India. She is currently pursuing her $\mathrm{PhD}$ in the area of data mining at VIT Chennai. She also holds the Bachelor's and Master's degrees in computer science and engineering from Manonmanium Sundaranar University and Anna University, Chennai, respectively. So far, Ms Syed Azimuddin has published 4 research papers in various national, international journals and conferences.

KaLYANI DesiKan is a professor of mathematics in the School of Advanced Sciences at VIT Chennai, India. She has over 22 years of experience in teaching and research. She has been a member of the faculty team that was instrumental in establishing the Mathematics Laboratory for undergraduate Engineering students of VIT, where she teaches calculus and differential equations with specific emphasis on applications and visualization. Her research interests include data mining, clustering, link analysis, automata theory, spectral graph theory and cosmology. She has mentored one $\mathrm{PhD}$ student and is currently mentoring four $\mathrm{PhD}$ students. 